\title{
Delay Differentiation by Optimal-Balancing-of-Queue-Lengths Scheduling
}

\author{
AvijitChakraborty ${ }^{*}$, Rajarshi Roy ${ }^{\dagger}$, and Utpal Mukherji ${ }^{\dagger}$ \\ $\dagger$ Department of Electrical Conununication Engineering \\ Indian Institute of Science \\ Bangalore, 560012 WDIA \\ 080-293-2387 \\ avijitc@ece.iisc.emet.in, utpal@ece.iisc.emet.in \\ $\ddagger$ Department of $\mathbf{E} \&$ ECE \\ Indian Institute of Technology \\ Kharagpur 721302 INDIA \\ $03222-75-5221$ \\ royr@ece.iitkgp,ernet.in
}

Absiract-- Scheduling policies adopted for statistical multiplexing should provide delay differentiation between different traffic classes. where each class represents an aggregate traffic of individual applications having the same target queueing delay requirement. We propose scheduling to optimally balance queue lengths as an approach to delay differentiation.

In particular, we assume a discrete-time, two-class, singleserver queueing model with unit service time per packet. We find 3 scheduling policy that we show to be discounted-cost optimal, for Bernoulli packet arrivals using dynamic programming analysis and for i.i.d. batch arrivals using a stepwise cost-dominance analyticalapproach. We then use cumulative queue length state variables in the one-step cost function of the optimization formulationandohtain the next-stage optimal policy. Simulations show that this policy achieves long-term mean queueing delays closer to the respective target delays than the first policy, and also achieves smaller errors in short-term mean queueing delays than the CoffmanMitrani policy.

\section{INTRODUCTION}

In scenarios such as wnununication via satellite, where signal propagation delays are large. the queueing delay margins available to packet communication applications can be small [1]. Further, these margins can be different for different applications. Hence. when packets from different applications are multiplexed statistically and transmitted on a single shared channel, the packets should be classified according to their delay requirements and scheduled for transmission using a suitably designed class-based scheduling policy.

Given the packet arrival statistics for different classes, it is possible to determine whether it is feasible to attain mean class queueing delays within their respective delay margins $[2,3]$. Having chosen a feasible set of mean class queueing delays. it remains to determine a scheduling policy that will achieve delays that are dose to the larget mean delays when averaged over time intervals of sufficient length

The Coffman-Mitranipolicy in [2] exactly achieves a feasible set of target long-term mean queueing delays, but the shortterm performance may be poor. Subsequent approaches that have been reported either are primarily heuristic in approach [3], or requires somewhat involved computations to obtain policy parameters $[4]$.

Orr approach is to formulate a weighted-quene-lengthbalancing optimization problem in the framework of Marknv decision theory. where class weights are set inversely proportional to the respective products of target delays and packet arrival rates. For the sake of simplicity, we assume that the class weights are identical (set to 1), i.e., the target delays are inverselyproportional to the arrival rates of the respective classes. If instantaneous queue lengths could be equalized by a scheduling policy then the mean queueing delays achieved by the policy would equal the respective target delays: Although exact instantaneous equalization is not possible, the mean queueing delays achieved by a policy that attempts to equalize cumulative queue lengths ma be close to the respective target delays.

The rest of the paper is organized as follows. In Section 2 , we describe a multiplexing model and analytically obtain an optimal policy for Bernoulli packet arrivals and for i,i.d. batch arrivals. In this section, we also use cumulative queue length state variables in the optimization problem and obtain the next-stage optimal policy. In Section 3, simulation results are presented for the above policies as well as for the Coffman-Mitrani policy, indicating the errors in the shortterm and long-term mean queueing delays relative to the target delays, and the relative values of the coefficients of variation for queueing delays. Section 4 concludes the paper. 


\section{TENCON $2003 / 1220$}

\section{Modeling AND ANALYSIS}

A survey of numerous Markov decision models, proposed for applications in communication networks, can be found in [5]. In [6], a two-class multiplexer has been modeled as a discretetime single-server two-quelie system with unit service time per packet and i.i d. packet hatch arrivals to each input queue (figure 1).

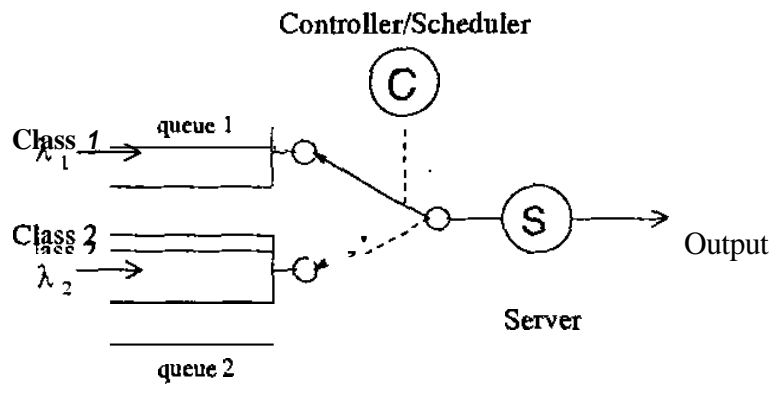

Figure 1. A two-class multiplexer

\section{Bernoulli Arrivals}

For the same model specialized to the case of Bernoulli arrivals to each input queue (arrival rate $\lambda_{i}$ to queue i), we formulate an optimal scheduling problem with total discounted cost minimization criterion associated with a one-step cost function of the two queue length state variables. The cost function is taken to be symmetric in the two co-ordinates, uondecreasinp in each co-ordinate, and increasing with modulus of difference between co-ordinates for fixed sum of coordinates. Examples of such one-step cost functions are $C(i, j)=i^{p}+j^{p}$ for $r>1, C(i, j)=\max (i, j)$, and $C(i, j)=b^{i}+b$ for $b>2$.

The total expected discounted cost over $N$ stages under policy $\pi$, for discount factor $\beta \in(0,1]$ and initial state $\tilde{x}$, is given by the value function

$$
\begin{aligned}
& V_{N}^{\pi}(\tilde{x})=E^{\pi}\left[\sum_{k=0}^{N-1} \beta^{k} C(\tilde{X}(k)) \mid \tilde{X}(0) \quad x\right], \forall N \geq 1 ; \\
& V_{0}^{\pi}(\tilde{x}):=0 \\
& \text { where } \bar{X}(k) \text { denotes the state at time } k
\end{aligned}
$$

Let $\Pi$ be the set of all policies. We want to find $\pi^{*} \in \Pi$, such that $V_{N}^{\pi^{*}}(\tilde{x})=\inf _{-\in \Pi} V_{N}^{\pi}(\tilde{x})$. This optimal value function is denoted by $V_{N}(\tilde{x})$.

The Dynamic Programming results for finite action space $U($ i) [7] yield the following optimality equation:

$V_{N}(\tilde{x})=C(\tilde{x})+\beta \min _{\bar{u} \in \mathcal{U}(\tilde{x})}\left[\sum_{\tilde{x}^{\prime}} P_{\tilde{x} \bar{x}^{\prime}}(\bar{u}) V_{N-1}\left(\tilde{x}^{\prime}\right)\right], \forall N \geq 1$

where $P_{\tilde{x} \tilde{x}^{\prime}}(\tilde{u})$ is the controlled Markov chain state transition probability.
We solve for an optimal policy by proving some structural properties for $V_{N}(.,$.$) (Lemma 1$, stated in Appendix A.1) and obtain the following result.

Theorem I: An optimal policy, for each horizon length $N$, serves at each time $n$ the queue with index

$$
\arg \max _{i=1.2}\left\{X_{i}(n)+\lambda_{i}\right\}
$$

i.e., serves the longer queue when queue lengths are unequal, and a queue with the maximum arrival rate whenqueue lengths are equal.

We also note that, following this policy, the modulus of the difference of the individual queue lengths is atmost 2 at any time after the queue lengths are first equal.

\section{I.I.D.batch Arrivals}

The model with 1.1 . batch arrivals in successive time units was considered in [6] with specific one-step cost function equal to the sum of the squares of the queue lengths. It was shown in [6] using a step-wise cost-dominance analytical approach, that an optimal stationary policy, for each horizon length, serves the longer queue when queue lengths are unequal, and the queue with the maximum amval rate when queue lengths are equal and the arrival rates differ.

In the special case of identical arrival statistics for the two classes, we show that an optimal policy serves either one of the queues when both queue lengths are equal.

\section{One-Step Cost Function of Cumulative Queve Lengths}

So far, we have used only instantaneous queue lengths as state variables in our optimization prohlems. Simulations show that the ratios of the resulting long-term average queueing delays for the two queues are riot satisfactorily close to the ratios of the target delays. We seek an improvement hy assuming a one-stepcost function equal to the sum of squares of cumulative queue lengths, The state at time $\mathrm{n}, X(n)$, is given hy the four-tuple $\left(X_{1}(n), X_{2}(n), \sum_{k=0} X_{1}(k), \sum_{k=0}^{n} X_{2}(k)\right)$. Then the one-step cost function at time $n$. is

$$
\begin{aligned}
& C(\tilde{X}(n))=\left(\sum_{k=0}^{n} X_{1}(k)\right)^{2}+\left(\sum_{k=0}^{n} X_{2}(k)\right)^{2} \\
& =\frac{1}{2}\left[\left(\sum_{k=0}^{n} X_{1}(k)-\sum_{k=0}^{n} X_{2}(k)\right)^{2}+\left(\sum_{k=0}^{n} X_{1}(k)+\sum_{k=0}^{n} X_{2}(k)\right)^{2}\right]
\end{aligned}
$$

We note that the sum $X_{1}(k)+X_{2}(k)$, at any time $k$, is identical for all work-conserving policies. Hence, with the optimization restricted to the space of work-conserving policies, this choice for $C(\tilde{X}(n))$ is an attempt to equalize cumulative queue lengths.

We obtain the following next-stage optimal policy (i.e., with $N=2$ ). 


\section{Packet-Switched Networks / 1221}

Theorem 2: The next-stage optimal work-conserving policy serves, at each time $n$ for which $X_{1}(n) \neq 0$ and $X_{2}(n) \neq 0$, the queue with index

$$
\arg \max _{i=1,2}\left\{\sum_{k=0}^{n} X_{i}(k)+X_{i}(n)+\lambda_{i}\right\},
$$

i.e., serves the queue with higher value of the sum of cumulative queue length. instantaneous queue length, and arrival rate. If the sums for both queues are equal, serving either queue is optimal.

\section{Simulation Results}

In this section, we discuss the performance of the optimal policy using instantaneous queue lengths, the next-stage optimal policy using cumulative queue lengths, and the CoffmanMitrani policy [2].

We firstbriefly describe the Coffman-Mitrani policy for our assumed model. Let $S_{1}$ and $S_{2}$ he two policies giving strict priority to class 1 and class 2 packets respectively. Then a mixing policy is constructed which, at the beginning of each busy period, decides with probability $a$ that the scheduling decisions during that busy period are to be made according to $S_{1}$, and with probability $1-\alpha$ the decisions are to be made according to $S_{2}$. Given the arrival statistics, the probability $\alpha$ is a constant and is determined solely by the target mean queue length of either class. Let $\bar{X}_{1}^{1}$ and $\bar{X}_{1}^{2}$ he the mean queuelengths of class I under policies $S_{1}$ and $S_{2}$ respectively. For all work-conservingpolicies, the sum of the mean queue lengths of both the classes is identical and denoted by $\bar{X}_{\text {total }}$ Because of the equal class weight assumption. the target mean queue length of class 1 is $\bar{X}_{1}^{t}=\bar{X}_{\text {total }} / 2$. Then,

$$
\alpha=\frac{\bar{X}_{1}^{2}-\bar{X}_{1}^{t}}{\bar{X}_{1}^{2}-\bar{X}_{1}^{1}}
$$

For all the cases below.

- we have simulated a total of 36 uniformly distributed arrival rate pairs at total arrival rates from 0.2 upto 0.9 , in increments of 0.1 in the individual class arrival rates,

- long-term average queueing delays are taken over a fixed length of $10^{6}$ time units,

- short-term average queueing delays are taken over 1000 windows of length 1000 time units each,

- average over different arrival rate pairs refers to the arithmatic average over feasible arrival rate pairs alone (i.e., those pairs for which the ratio of the target mean queueing delays, which is determined as the reciprocal of the ratio of arrival rates because of the equal class weight assumption, is feasible given the arrival statistics).

\section{Bernoulli Arrivals}

Irrespective of the policy adopted, the number of feasible arrival rate pairs is 23 . out of the total of 36 amval rate pairs.
Using Instantaneous Queue Lengths, the ratios of the resulting long-term average queueing delays are within $25 \%$ of the ratios of the target delays for 18 out of 23 feasible arrival rate pairs. The average of the errors in the ratio of long-term average delays is approx. $21 \%$ and the average of the errors in the ratio of short-tenn average delays is also approx. $21 \%$. For the arrival rate pair $(0.3,0.4)$, the error in the ratio of longterm average delays is approx. $25 \%$, and the errors in the ratios of short-term average delays are also $25 \%$ on average, the maximum value over 1000 windows being approx. $42 \%$. These results are improvements upon the performance of the serve-the-longest-queue policy.

Using Cumulative Queue Lengths, the ratios of the resulting long-term average queueing delays are within $0.71 \%$ of the ratios of the target delays forall feasible arrival rate pairs. and within $0.31 \%$ of the ratios of the target delays for 18 out of the 2.3 feasible arrival rate pairs. The average of the errors in the ratio of long-term average delays is approx. $0.2 \%$, while the average of the errors in the ratio of short-term average delays is approx. $6 \%$. For the amval rate pair $(0.4,0.2)$, the error in the ratio of long-term average delays is approx. $0.31 \%$.but the errors in the ratios of short-term average delays are $7 \%$ on average, the maximum value over 1000 windows being approx. $26 \%$.

If the Coffman-Mitrani policy is employed. the ratios of the resulting long-term average queueing delays are within $1 \%$ of the ratios of the target delays for 18 out of the 23 feasible arrival rate pairs. The average of the errors in the ratio of long-term average delays is approx. $0.7 \%$, but the average of the errors in the ratio of short-term average delays is approx. $25 \%$. For the arrival rate pair $(0.1,0.8)$, the error in the ratio of long-term average delays is approx. $1 \%$. hut the errors in the ratios of short-term average delays are $21 \%$ on average, the maximum value over 1000 windows being approx. $151 \%$.

\section{I.I.D. Batch Arrivals with Poisson Butch Sizes}

Irrespective of the policy adopted, the number of feasible ar rival rate pairs is 22 , out of the total of 36 arrival rate pairs.

Using Instantaneous Queue Lengths, the ratios of the resulting long-term average queueing delays are within $30 \%$ of the ratios of the target delays for 16 out of 22 feasible arrival rate pain. The average of the errors in the ratio of long-term average delays is approx. $22 \%$ and the average of the errors in the ratio of short-term average delays is approx. $24 \%$. For the anival rate pair $(0.2,0.6)$, the error in the ratio of long-term /average delays is approx. $30 \%$, and the errors in the ratios of short-tenn average delays are also $30 \%$ on average, the maximum value over 1000 windows heing approx. $48 \%$.

Using Cumulative Queue Lengths. the ratios of the resulting long-term average queueing delays are within $0.59 \%$ of the ratios of the target delays for all feasible arrival rate pairs, and within $0.25 \%$ of the ratios of the target delays for 16 out of the 22 feasible arrival rate pairs. The average of the errors 
in the ratio of long-term average delays is approx. $0.2 \%$. while the average of the errors in the ratio of short-term average delays is approx. $10 \%$. For the arrival rate pair $(0.4,0.4)$, the error in the ratio of long-term average delays is approx. $0.25 \%$, hut the errors in the ratios of short-term average delays are $6 \%$ on average, the maximum value over 1000 windows being approx. $25 \%$.

If the Coffman-Mitrani Policy is employed, the ratios of the resulting long-term average queueing delays are within $1 \%$ of the ratios of the target delays for 16 out of the 22 feasible arrival rate pairs. The average of the errors in the ratio of long-term average delays is approx. $0.8 \%$.hut the average of the errors in the ratio of short-term average delays is approx. $43 \%$. For the arrival rate pair $(0.4,0.5)$, the error in the ratio of long-term average delays is approx. $1 \%$, hut the errors in the ratios of short-term average delays are $102 \%$ on average, the maximum value over 1000 windows being approx. $1659 \%$.

We note that apart from improved long-term average behaviour, using cumulative queue lengths results in improved short-term average behaviour as well, as shown in figures 2 and $\mathbf{3}$ (drawn in log-log scale).

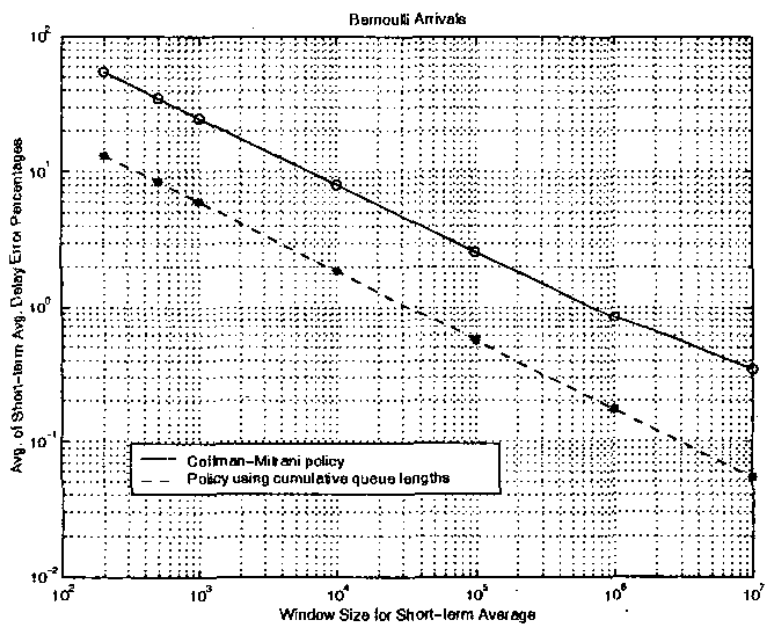

Figure 2. Comparison of short-term behaviour for Bernoulli arrivals

\section{Coefficients of Variation of Queueing Delays}

Another criterion of importance is the coefficient of variation (ratio of standard deviation to mean) of queueing delay. Let $c_{i}, c_{c}$, and $c_{r}$ denote the coefficients of variation of the queueing delays of any class for the policies using instantaneous queue lengths, using cumulative queue lengths, and using the Coffman-Mitrani policy respectively.

For feasible Bernoulli amval rate pairs, the values of $c_{i}, c_{c}$, and $c_{2 \pi}$ are in the respective ranges $0.04-0.90,0.00-0.90$, and $0.00-1.30$. And the differences $c_{c}-c_{i}, c_{m}-c_{i}$, and $c_{m}-c_{c}$ vary between -0.27 and $0.24,-0.30$ and 0.50 , and -0.11 and 0.43 respectively.

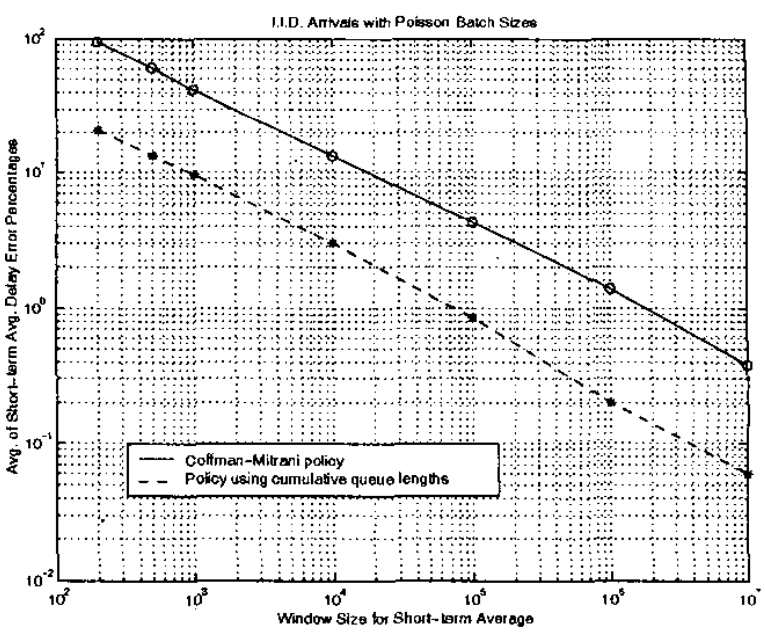

Figure 3. Comparison of short-term behaviour for i.i.d. batch arrivals with Poisson hatch sizes

And for i.j.d. batch arrivals with Poisson batch sizes, the values of $c_{i}, c_{c}$, and $c_{m}$ areintherespectiveranges $0.25-1.22$. $0.32-1.27$, and $0.32-1.54$. And the differences $c_{c}-c_{i}$, $c_{m}-c_{i}$, and $c_{m}-c_{c}$ vary between -0.12 and 0.11 . -0.12 and 0.55 , and -0.02 and 0.52 respectively.

\section{CONCLUSION}

The next-stage optimal policy obtained using cumulative queue length state variables in the one-step cost function of the optimization formulationachieves long-term mean queueing delays closer to the respective target delays than by using only instantaneous queue length state variables. This policy using cumulative queue lengths also achieves short-term mean queueing delays closer to the respective target delays than the Coffman-Mitrani policy.

Extensionsof this work to arbitrary class weights, larger number of classes, and correlated arrivals, and comparisons with the policies in $[3,4]$, are desirable.

\section{APPENDIX}

\section{A.l Lemma 1}

The three properties of the one-step cost function assumed for the Bernoulli arrival case in Section 2 are as follows:

$$
\begin{aligned}
& \text { Property A: } \quad C(i, j)=C(j,) \\
& \text { Property B : } \quad C(i+1, j) \geq C(i, j) \text { if } i \geq j \text {, } \\
& C(i, j)+1 \geq C(i, j) \text { if } \mathrm{j} \geq i \\
& \text { Property } \mathrm{C}: \text { if } i_{1}+j_{1}=i_{2}+j_{2},\left|i_{1}-i_{2}\right|=\mathbf{1} \text {, } \\
& \text { and }\left|i_{1}-j_{1}\right|>\left|i_{2}-j_{2}\right| \text {, } \\
& \text { then } C\left(i_{1}, j_{1}\right)>C\left(i_{2}, j_{2}\right)
\end{aligned}
$$

Starting with these properties, as $V_{1}(.$.$) is C(.,$.$) , we prove$ the following lemma by induction. 


\section{Packet-Switched Networks / 1223}

Lemma I: The $N$-stage optimal value function $V_{N}(.,),. \forall N$, has the following structural properties :-

(PI) if i $\geq \mathbf{j}+\mathrm{I}$, then $V_{N}(i, j) \geq V_{N}(i-1, j$ it !

(equality holds if $i=j+1$ );

(P2) if $\mathbf{j} \geq i+1$, then $V_{N}(i, j) \geq V_{N}(i+1, j-1)$

(equality holds if $j=i+\mathrm{I}$ );

(P3) if $i=j$, then $V_{N}(i+1, j-1) \geq=\leq V_{N}(i-1, j)+1$ according as $\lambda_{1}>=<\mathrm{A} ;$;

(P4) $V_{N}(i, \mathbf{j})$ is non-decreasing in each co-ordinate.

\section{A.2 Proof of Theorem 2}

Lct the state at time $n, \tilde{X}(n)=\left(X_{1}(n), X_{2}(n), \sum X_{1}(k)\right.$, $\left.\sum X_{2}(k)\right)$, be $(i, j, s, t), i, j \neq 0$.

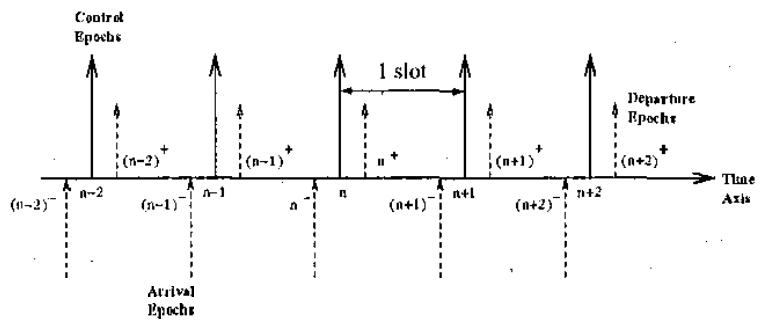

Pigurc 4. Timing Diagram

Departure occurs just alter a controlepoch, and arrivals occur just prior to a control epoch (figure 4).

Then, if queue I is served at time $n, \tilde{X}(n)+1$ will bc

$\left(i-1+A_{1}(n)+1, j+A_{2}(n)+1, s+i-1+A_{1}(n)+1\right.$, $t+j+A_{2}(n)+1$,

and if queue 2 is served $n t$ time $n, \tilde{X}(n)+1$ will be

$\left(i+A_{1}(n)+1, j-1+A_{2}(n)+1, s+i+A_{1}(n)+1\right.$,

$t+j-1+A_{2}(n)+1$,

where $A_{m}(n)+1$ form $=1,2$ denote the arrivalsjust prior to time $n+1$ (figure 2) to queue 1 and queue 2 respectively.

From the choice of the ond-step cost function, and denoting the cost incurred at time $n+1$ when queue $m$ is served at time $n$ by $C^{m}(n)+1, s+i+A_{1}(n)+\mathrm{I}$ by $P_{1}$, and $t+j+A_{2}(n)+1$ by $P_{2}$, we have

$$
\begin{aligned}
& E\left[C^{\mathbf{l}}(n)+1 \mid \tilde{X}(n)=\left(i, \xi_{n}\right)\right] \\
= & E\left[\left(P_{1}-1\right)^{2}+P_{2}^{2} \mid \tilde{X}(n)=\left(i, \xi_{n}\right)\right], \\
& E\left[C^{2}(n)+1 \mid \tilde{X}(n)=\left(i, \xi_{n}\right)\right] \\
= & E\left[P_{1}^{2}+\left(P_{2}-1\right)^{2} \mid \tilde{X}(n)=\left(i, \xi_{m}\right)\right] .
\end{aligned}
$$

$$
\text { Hcncc, } \begin{aligned}
& E\left[C^{\mathrm{L}}(n)+\mathrm{L}-C^{2}(n)+1 \mid \tilde{X}(n)=\left(i, \phi_{n}\right)\right] \\
&= E\left[\left\{P_{2}^{2}-\left(P_{2}-1\right)^{2}\right\}-\left\{P_{1}^{2}-\left(P_{1}-1\right)^{2}\right\}\right. \\
&\left.\mid \tilde{X}(n)=\left(i, \xi_{n}\right)\right] \\
&= 2 E\left[\left\{P_{2}-P_{1}\right\} \mid \tilde{X}(n)=\left(i, \hbar_{n}\right)\right] \\
&= 2\left[\left\{l+j+\lambda_{2}\right\}-\left\{s+i+\lambda_{1}\right\}\right]
\end{aligned}
$$

The next-stageoptimal policy serves queue 2 / either cueue / queuc 1 according as

$E\left[C^{1}(n)+1-C^{2}(n)+1 \mid \tilde{X}(n)=\left(i, z_{n} \quad\right)\right]>=<0$, i.e., according as $\left\{t+j+\lambda_{2}\right\}>=<\left\{s+i+\lambda_{1}\right\}$.

We note that lor Markovian arrival processes, the state of the arrival process lass to be included in the state clescription of the system, and $\lambda_{1}$ and $\lambda_{2}$ will be arrival state dependent.

Replacing $i, \mathbf{j}, s$, and $t$ by $X_{1}(n), X_{2}(n), \sum_{k=1} X_{1}(k)$, and $\sum_{k=0} X_{2}(k)$ respectively, Theorem 2 is proved.

\section{REFERENCES}

[1] F. Gargione, T. Iida, F. Valdoni, and F. Vatalaro, "Selvices, Technologies, and Systems at Ka Band and Beyond - A Survey," IELLL.I. Selected he n s in Communications, vol. 17, no. 2, pp. 133-144, Fcb. 1999.

[2] E. G. Coffinan. Jr. and I. Mitrani, "A Characterization of Waiting Time Performance Realizable hy SingleServer Queues:' Operation Research, vol. 28, no. 3, part II, pp. 810-821, May-June 1980.

[3] C. Dovrolis, D. Stiliadis, and P. Ramanathan, "Proportional Differentiated Services: Delay Diflerentiation and Packet Sohcduling," WEEL, ACM Tram. Networking, vol. 10, no. I, pp. 12-26, Feb. 2002.

[4] M. K. H. Leung, 3. C. S. Lui, and D. K. Y. Yau, "Adaptive Proportional Delay Differentiated Services: Characterization and Performance Evaluation," $\mathrm{LLWE} / \mathrm{ACM}$ Thans. Networking, vol. 9, no. 6, pp. 801-817, Dec. 2001.

[5] E. Altman, "Applicationsol Markov D xision Processes in Communication Networks," Handbook of Markov" Decision Processes (E. A. Feinberg and A. Sliwart? Ed.), Kluwer Academic, 2002.

[6] R. R. Pillai, and U. Mukherji, "Optimal Scheduling for Balaneing Bulfer Occupancy at an ATM Acccss Point:' Proceedings: International Conference on Information. Communications and Signal Processing, EEE, Singapore, pp. 649-653, Scpt. 1997.

[7] D. P. Bcrtsckas, Dynamic: Programming and Optimal Control, vol. 1, Athena Scientific. 1995. 